\title{
Serial position effects in recognition memory for odors: A reexamination
}

\author{
CHRISTOPHER MILES and KATHRYN HODDER \\ Cardiff University, Cardiff, Wales
}

\begin{abstract}
Seven experiments examined recognition memory for sequentially presented odors. Following Reed (2000), participants were presented with a sequence of odors and then required to identify an odor from the sequence in a test probe comprising 2 odors. The pattern of results obtained by Reed (2000, although statistically marginal) demonstrated enhanced recognition for odors presented at the start (primacy) and end (recency) of the sequence: a result that we failed to replicate in any of the experiments reported here. Experiments 1 and 3 were designed to replicate Reed (2000), employing five-item and seven-item sequences, respectively, and each demonstrated significant recency, with evidence of primacy in Experiment 3 only. Experiment 2 replicated Experiment 1, with reduced interstimulus intervals, and produced a null effect of serial position. The ease with which the odors could be verbally labeled was manipulated in Experiments 4 and 5. Nameable odors produced a null effect of serial position (Experiment 4), and hard-to-name odors produced a pronounced recency effect (Experiment 5); nevertheless, overall rates of recognition were remarkably similar for the two experiments at around 70\%. Articulatory suppression reduced recognition accuracy (Experiment 6), but recency was again present in the absence of primacy. Odor recognition performance was immune to the effects of an interleaved odor (Experiment 7), and, again, both primacy and recency effects were absent. There was no evidence of olfactory fatigue: Recognition accuracy improved across trials (Experiment 1). It is argued that the results of the experiments reported here are generally consistent with that body of work employing hard-to-name visual stimuli, where recency is obtained in the absence of primacy when the retention interval is short.
\end{abstract}

The extent to which the results of tests of memory for olfactory stimuli resemble those for other modalities, in terms of both their pattern and their reliability, is equivocal. Indeed, Herz and Engen (1996) conclude that the current lack of data precludes any conclusions with respect to both the shape and the duration of short-term olfactory memory. However, one area in which there appears to be a striking contradiction in the literature concerns the effect of serial presentation of odors on immediate recognition for those odors. In a typical recognition task, the participant receives a sequence of items followed by two test items, one of which is familiar, and the participant is required to identify the familiar item. This is referred to as a two-alternative forced choice (2AFC) task (see e.g., Reed, 2000). Variations on this form of recognition task in the visual domain using sequences of novel, hardto-name stimuli with immediate testing (e.g., unfamiliar faces, digitized snowflakes) generally produce recency effects in the absence of primacy effects (Avons, Ward, \& Melling, 2004; Kerr, Ward, \& Avons, 1998; Neath, 1993;

The authors thank Bryony Berry, Paul Allen, and Anna Biles for their help with data collection, Andrew Johnson for statistical advice, Rob Honey and Simon Killcross for their useful discussions concerning this work, and Geoff Ward and two anonymous referees for their helpful comments on an earlier version of this paper. Correspondence should be addressed to C. Miles, School of Psychology, Cardiff University, Cardiff CF10 3AT, Wales (e-mail: miles@cardiff.ac.uk).
Phillips \& Christie, 1977; Ward, Avons, \& Melling, 2005). This particular pattern of recognition was originally established by Phillips and Christie (1977), using visually presented patterns formed by randomly filled cells in a $4 \times$ 4 matrix. Using a same-different test with reverse testing of serial order, they reported a recognition rate of $96.9 \%$ for the last pattern in the sequence. In contrast, recognition for earlier items was lower (although above chance) at $62.6 \%$. The latter result was interpreted as reflecting the action of a stable long-term memory component with the capacity to represent many patterns, whereas the recency effect was taken to reflect the action of a singleitem short-term memory store. Thus, the data were nicely encapsulated within a two-stage information-processing model. It is worthwhile to note, however, that these recognition tasks typically employ tests of novel items tested in reverse order; that is, the most recently presented item is tested first, and subsequent testing proceeds backward through the list.

In contrast to the results of studies employing unfamiliar, hard-to-name visual stimuli, Reed (2000), using sequences of odors, reported the results of a series of $2 \mathrm{AFC}$ recognition memory experiments that consistently demonstrated both recency and primacy effects. However, other experiments employing sequences of odors (e.g., White, 1992; White \& Treisman, 1997, Experiment 3), in contrast to Reed (2000), provide evidence for olfactory recency in the absence of olfactory primacy. Annett and Lorimer 
(1995) presented sequences of 17 odors accompanied in one condition with instructions to verbally elaborate the stimuli and in another condition without such instructions. Although recency was evident in both conditions, primacy was present in the verbal elaboration condition only. Similarly, Miles and Jenkins (2000) reported a series of experiments in which participants were initially trained to label verbally a series of olfactory stimuli. Participants were then presented with these stimuli in an immediate serial recall task. The recall profiles demonstrated both recency and primacy effects. Critically, recency was greatly attenuated for those sequences followed by a to-be-ignored olfactory suffix, but recency was immune to the effects of both an auditory and a visual suffix. The overwriting effect of the same-modality suffix is consistent with the Phillips and Christie (1977) proposal of a fragile, single-item store that represents the terminal list item. To this extent, the pattern of data reported by Miles and Jenkins is typical of that found when auditory verbal materials are employed (although see Surprenant, Pitt, \& Crowder, 1993). However, the implicit assumption made by Miles and Jenkins, unlike Reed (2000), is that primacy reflected rehearsal of the verbal labels associated with each list odor. The Miles and Jenkins experiments are therefore limited in the extent to which they provide evidence concerning both the storage and the representation of preterminal olfactory stimuli in immediate memory when the opportunity for verbal mediation is much reduced. Indeed, the extent to which spontaneous verbal mediation processes underpin human olfactory memory remains contentious (see Herz \& Engen, 1996, for a review).

To date, the only recognition study with respect to olfactory sequences in human participants that demonstrates both primacy and recency appears to be that reported by Reed (2000). As Reed (2000) acknowledges, the demonstration of primacy in his experiments (at least Experiments 1-3) might be underpinned by a process whereby participants assigned verbal labels to the list items. Notwithstanding Reed's (2000) observation that participants were generally very poor at verbally labeling the experimental odors at the completion of Experiment 3 , the extent to which verbal processing was evident remains contentious. The problematic issue of language processing mechanisms underpinning studies concerned with serial position effects in odor recognition is negated by testing nonhuman animal participants. As suggested by White (1998), given the salience of odors in the lives of most animals, it is quite plausible that the order in which odors are perceived is of importance. However, evidence for a full serial position curve (i.e., the presence of both primacy and recency) for odor recognition in rats is equivocal. For instance, data by Reed, Chih-Ta, Aggleton, and Rawlins (1991) using a nonmatching-to-sample procedure evidencing both primacy and recency effects were subject to statistical criticism (Gaffan \& Gaffan, 1992) that pointed to the less-than-expected variance in the data, in particular at Serial Position 3 (the midpoint) of the serial position curve. Rawlins, Deacon, Tai, and
Aggleton (1992) examined olfactory memory in rats with a nonmatching-to-sample procedure, in an attempt to replicate the earlier Reed et al. (1991) findings. Rats were presented with five odors and then required to select one of two odor-containing boxes. One box contained a novel odor, and the other box contained an odor from the previously presented sequence. The results showed a bias toward correctly selecting the novel odor when paired with an odor presented more recently in the original sequence: that is, recency in the absence of primacy. (For a full discussion, see also Gaffan, 1992, 1994; Kesner, Chiba, \& Jackson-Smith, 1994; Reed, 1992, 1994; and Wright, 1994.) Reliable recency data have been demonstrated with insects (Leptophilina boulardi, a parasitic wasp of Drosophila larvae). Insects were preexposed to three odorants: Banana, Strawberry, and Violet. Order of learning was assessed by preference for particular odors. The most recently presented (third) odor was preferred over the second learned odor, suggesting greater familiarity for that odor relative to those presented earlier (Kaiser \& De Jong, 1993).

\section{EXPERIMENT 1}

Given the apparent uniqueness of Reed's (2000) results with human participants, Experiment 1 in the present series was designed to assess the reliability of Reed's (2000, Experiment 1) data by replication. In this respect, our experimental design replicated that of Reed (2000) directly, and, to improve the power of the experiment, the number of participants was doubled to $N=24$. Two of the seven odors employed by Reed were no longer available and were therefore substituted with very similar odors. These substitutions were made on the advice of The Body Shop staff.

\section{Method}

Participants. Twenty-four psychology undergraduates (20 females, 4 males; mean age $=22$ years 10 months) from Cardiff University participated in exchange for either course credit or payment.

Materials. Five of the olfactory stimuli were the same as those employed by Reed (2000, Experiment 1 ). Two of the odors had to be replaced with close approximations, since they were no longer available at The Body Shop. The complete set of odors was White Musk, Potpourri, Oceanus, Vanilla, Spirit of Moonshine (to replace Ananya), Blackcurrant (to replace Dewberry), and Strawberry. Each odor was presented as a liquid soaked into cotton wool contained in a $20-\mathrm{ml}$ brown glass bottle. Each bottle had an identical visual appearance. The bottles were held in a wooden rack behind a wooden screen and were thereby shielded from the view of the participant. These odors were tested for discriminability with 2 participants, who were each presented with odor pairs. Each odor was presented with every other odor and itself, and the participants made a samedifferent forced choice judgment for each pair. The mean percentage of correct same judgments was $99 \%$, and the mean percentage of correct different judgments was $95 \%$, confirming the discriminability of the odors.

Design. The design followed that described by Reed (2000). A within-participants design was adopted in a $2 \mathrm{AFC}$ decision paradigm. Four blocks each of five trials were prepared. Within each block, each serial position was tested once in a random order, thereby representing a complete determination for the serial position function. Across the four blocks, each of the seven odors occurred an 
approximately equal number of times at each serial position in the list and an equal number of times as either the same stimulus or a different stimulus in the recognition test. The order of block presentation was randomized across participants.

Procedure. The procedure followed that reported by Reed (2000). Each participant was tested individually in a well-ventilated soundproof experimental laboratory and sat facing the experimenter with a fan blowing directly across his/her face. The participant sat approximately $50 \mathrm{~cm}$ from a wooden screen and was unable to see the experimental materials. The participant was instructed to fixate on a red spot $15 \mathrm{~cm}$ in front of the screen throughout the experiment. In each trial, the participant was presented with a series of five odors. Each odor was passed over the center of the screen and presented under the participant's nose. The participant was exposed to each odor stimulus for approximately $3 \mathrm{sec}$, during which time the participant inhaled. The odor was then replaced behind the screen, during which time the participant exhaled. The next odor was presented to the participant with a minimum delay of approximately $2 \mathrm{sec}$.

A retention interval of $3 \mathrm{sec}$ followed the sequential presentation of the five odors. The participant was then presented sequentially with two test odors, one identical to and the other different from one of the odors in the immediately preceding sequence. These test odors were presented at the same rate as that of the list odors. After presentation of the second test odor, the participant was required to indicate verbally (i.e., "first" or "second") which of the two odors was familiar. Each trial was separated by an interval of approximately $5 \mathrm{sec}$, and each block was separated by an interval of approximately $2 \mathrm{~min}$.

\section{Results and Discussion}

Figure 1 shows the mean percentage of correct item recognition for each serial position. The participants recognized odors more accurately when they were presented at the end of the list than when they were presented in the middle or at the start of the list. An analysis of variance (ANOVA) with serial position as a repeated measures factor was conducted on the recognition data. A rejection criterion of $p<.05$ was adopted for this and all subsequent analyses. The analysis just failed to achieve significance $\left[F(4,92)=2.27, M S_{\mathrm{e}}=0.83, p=.067\right]$. Trend analysis revealed a significant linear component $[t(23)=2.77$, $p<.05]$, reflecting the recency component of the slope. The quadratic component, however, failed to achieve significance $[t(23)=1.37]$, reflecting the lack of a full serial position curve.

Although the pattern of data for serial recognition demonstrates a clear failure to replicate the primacy component of the serial position curve as obtained by Reed (2000), its demonstration of recency in the absence of primacy is consistent with the results of a number of studies employing hard-to-name visual stimuli and short retention intervals (e.g., Kerr, Avons, \& Ward, 1999; Neath, 1993; Phillips \& Christie, 1977). Note, however, that the mean levels of recognition across serial position for the present experiment (69\%) and Reed's (2000) Experiment $1(71 \%)$ are very similar, suggesting equivalence in perceived task difficulty. Why the patterns of serial recognition for the two experiments should be so different is unclear. Both experiments employed undergraduates at United Kingdom universities (London and Cardiff), and it appears unlikely, therefore, that population differences could account for the failure to replicate. During the review process, it was suggested that participant differences between the present experiment and Reed's Experiment 1 might be implicated in the failure to replicate. In particular, it was speculated that female participants have more experience at naming olfactory cues than do male participants, and this might promote serial position effects-specifically, primacy - via a rehearsal process. In the present experiment, this seems unlikely, because 20 of the $24(83 \%)$ participants were female and a full serial position curve was not obtained.

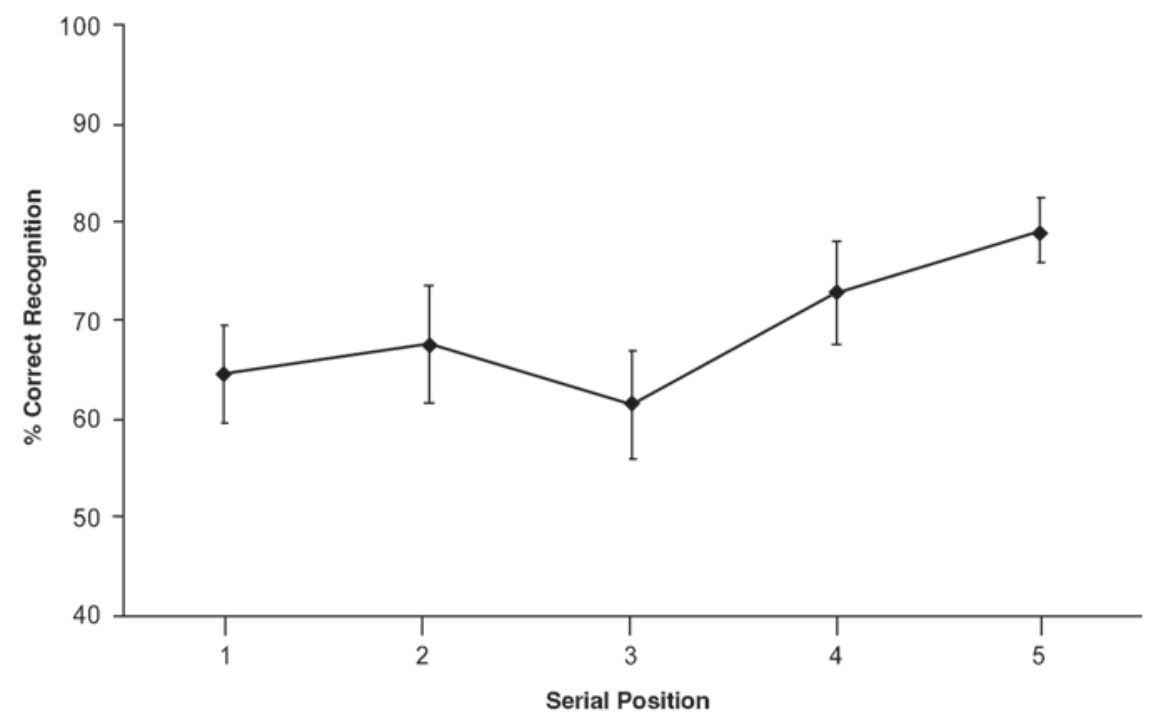

Figure 1. Percentage of odors correctly recognized, with standard error bars, at each serial position for Experiment 1. 
Following Reed (2000), the data were further analyzed for changes in both absolute recognition accuracy and the pattern of recognition accuracy across test trials via a comparison of recognition scores for the first block of trials with those of the final block of test trials (see Table 1).

The mean recognition rate across serial position was $72 \%$ for the first block of trials and $76 \%$ for the final block of trials (a $6 \%$ increase in recognition accuracy). This contrasts with the results of Reed (2000, Experiment 1), where the mean recognition rate across serial position was $74 \%$ for the first block of trials and $62 \%$ for the final block of trials (a 16\% decrease in recognition accuracy). Thus, recognition accuracy remained consistent across blocks in the present experiment but decreased across blocks in Reed's (2000) experiment. The data for the present experiment differ from those of Reed in another important respect. Reed's (2000) data show both primacy and recency effects for the first block of trials (recognition accuracy at Serial Positions 1 and 5 is above chance via a binomial test of significance). However, the recognition scores for these serial positions are nonsignificant for the final block of trials. That is, there is no evidence of either primacy or recency for the fourth block of trials. For the present experiment, binomial tests showed recognition accuracy to be above chance at Serial Position 5 in Block 1 and at Serial Positions 4 and 5 in Block 4. Thus, Experiment 1 demonstrated recency in the absence of primacy, and this pattern of recognition persisted across blocks. In summary, the present experiment failed to replicate Reed (2000, Experiment 1) in the following respects: (1) the overall pattern of serial recognition, (2) the change in recognition rate for early trials contrasted with later trials, and (3) the pattern of serial recognition for both early and late trials.

\section{EXPERIMENT 2}

The reason for the discrepancy between the pattern of data reported here and that of Reed (2000, Experiment 1) is unclear. However, it is possible that the lack of primacy in our Experiment 1 was due to the greater opportunity for odors presented early in the sequence to decay from memory relative to odors presented later in the sequence. Overall recognition accuracy was $65 \%$ at Serial Position 1 and $79 \%$ at Serial Position 5. Therefore, Experiment 2 was designed to replicate Experiment 1 in all respects with the exception of the odor presentation time. In Experiment 2, this was reduced from 3 to $1 \mathrm{sec}$. The duration of the pre-

Table 1

Number of Participants $(N=24)$ Correctly Identifying Odors at Each Serial Position in Block 1 and Block 4 in Experiment 1

\begin{tabular}{cccccc}
\hline & \multicolumn{5}{c}{ Serial Position } \\
\cline { 2 - 6 } Block & 1 & 2 & 3 & 4 & 5 \\
\hline 1 & 14 & 16 & 16 & 18 & 22 \\
4 & 16 & 20 & 16 & 20 & 22 \\
\hline
\end{tabular}

sentation component of a trial (i.e., for the five odors plus the five interstimulus intervals [ISIs]) was thus reduced from $26 \mathrm{sec}$ in Experiment 1 to $16 \mathrm{sec}$ in Experiment 2. (The retention interval of $3 \mathrm{sec}$ was constant across both Experiment 1 and Experiment 2.) Thus, the potential for odors presented early in the memory sequence to decay from memory prior to the recognition test was much reduced in Experiment 2.

\section{Method}

Participants. Twenty-four psychology undergraduates (15 females, 9 males; mean age $=22$ years 6 months) from Cardiff University participated in exchange for course credit or payment. None had participated in Experiment 1.

Materials. The olfactory stimuli were the same as those described for Experiment 1.

Design and Procedure. The design and procedure were the same as those described for Experiment 1 with the exception that the presentation duration for each odor was reduced from 3 to $1 \mathrm{sec}$.

\section{Results and Discussion}

Figure 2 shows the mean percentage of correct item recognition for each serial position. Visual inspection of Figure 2 does not suggest a serial position curve, and an ANOVA revealed a nonsignificant effect of serial position $\left[F(4,92)=1.3, M S_{\mathrm{e}}=0.76\right]$. Trend analysis confirmed that neither the linear component $[t(23)=0.57]$ nor the quadratic component $[t(23)=1.7]$ achieved significance.

Thus, the proposal that lack of primacy in Experiment 1 was due to the rapid decay of the sensory olfactory representations for items early in the sequence finds no support from this experiment. Therefore, we can conclude that a reduction of approximately $38 \%$ in the presentation time of the odor sequence does not translate to a concomitant increase in recognition rate for the odors. Although the recognition rate at Serial Position 1 (the primacy index) increased from 65\% in Experiment 1 to $72 \%$ in Experiment 2, this difference failed to materialize as primacy in Experiment 2.

\section{EXPERIMENT 3}

The first two experiments in the present series are striking in their failure to fully replicate the pattern of findings reported by Reed (2000, Experiment 1). Experiment 3 in the present series was designed as a direct replication of a further experimental manipulation reported by Reed (2000, Experiment 2). In Reed's experiment, participants were presented with seven, rather than five, odors in series. The same recognition task as described previously was employed. One group of participants $(n=10)$ received a list-recognition test interval of $3 \mathrm{sec}$, and the other received a list-recognition test interval of $30 \mathrm{sec}$. Although the results showed a nonsignificant interaction $(F=2)$ between retention interval and serial position, only the 3 -sec retention interval group showed significant primacy and recency effects. In order to examine the reliability of Reed's demonstration of both primacy and recency effects with longer list lengths, Experiment 3 in the present series 


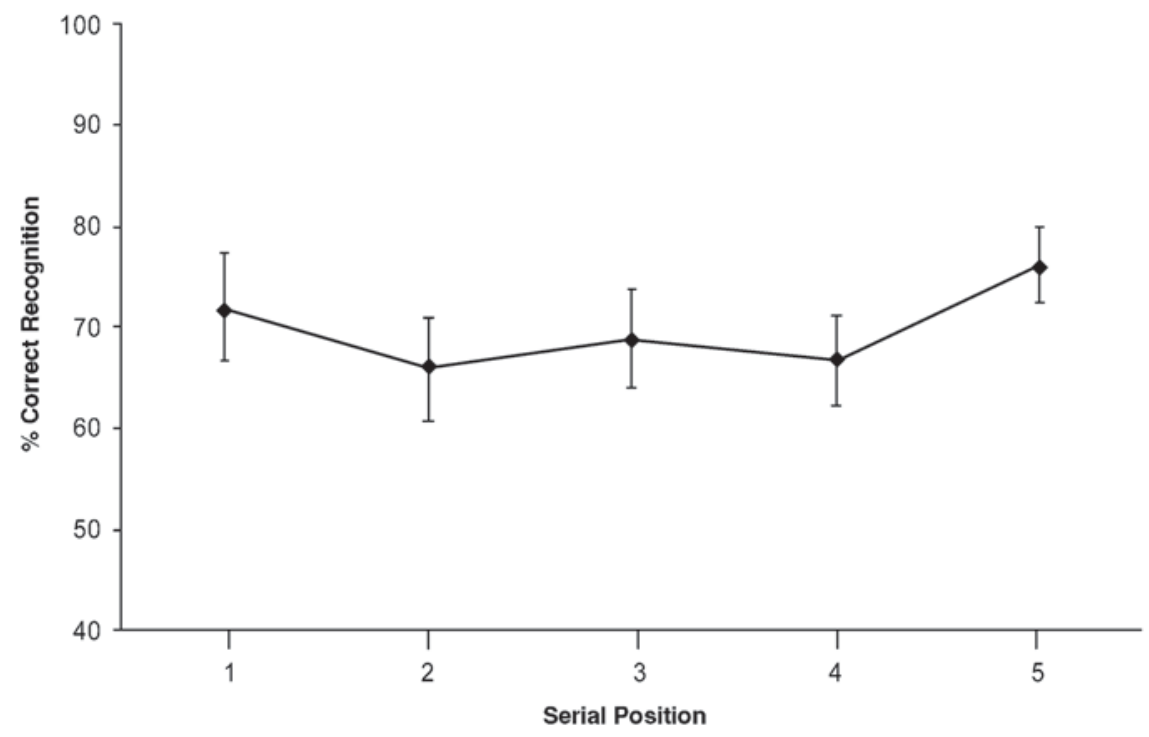

Figure 2. Percentage of odors correctly recognized at each serial position, with standard error bars, for Experiment 2.

was designed to replicate his 3 -sec retention interval condition with increased power $(N=24)$.

\section{Method}

Participants. Twenty-four psychology undergraduates (15 females, 9 males; mean age $=23$ years 11 months) from Cardiff University participated in exchange for course credit or payment. None had participated in the previous experiments.

Materials. The materials were the same as those described for Experiments 1 and 2 in the present series, with the addition of two more odors. The additional odors employed by Reed (2000) were no longer available from The Body Shop; therefore, Mixed Spice (to replace Woodland Spice) and Lavender (to replace Heartfelt) were introduced to the stimulus set.

Design. The design was the same as that described for Experiment 1 , with the exception that the odor list length was increased from five to seven items. Each block comprised seven trials; within each block, each serial position was tested once. Six blocks of trials were prepared, and each participant received a different random order of the six blocks. Within each block, the odors were all presented in each serial position and, as foil stimuli at test, approximately equal numbers of times, and each trial within a block randomly tested a different serial position.

Procedure. The procedure was the same as that described for Experiment 1.

\section{Results and Discussion}

Figure 3 shows the mean percentage of correct item recognition for each serial position and suggests superior recognition for items presented at the end of the list relative to those presented at the beginning.

A single-factor ANOVA conducted on the recognition data revealed an effect of serial position $[F(6,138)=7.51$, $\left.M S_{\mathrm{e}}=0.95, p<.001\right]$. Trend analysis revealed a significant linear component $[t(23)=5.87, p<.01]$, reflecting the recency element and, in line with Reed (2000, Experiment 3$)$, a significant quadratic component $[t(23)=$ 4.89], reflecting both primacy and recency. However, our data show much smaller primacy $(67 \%$ correct recognition at Serial Position 1, relative to $80 \%$ at this serial position in Reed's experiment). So, although both primacy and recency effects are statistically apparent by trend analysis for our data, the pattern of data does not constitute a true replication of the Reed data, notwithstanding the comparable recognition rates for the two experiments (51\% and 59\%, for Reed's experiment and the present experiment, respectively). Again, it is not obvious why the data between the experiments are discrepant in this way. Nevertheless, as in Experiment 1, the pattern of data here is consistent with that of experiments employing hard-toname, visual stimuli with immediate testing.

\section{EXPERIMENT 4}

The experiments reported thus far employed the stimuli selected by Reed (2000), and there is an unambiguous empirical discrepancy in the pattern of data produced in his laboratory and those produced in the Cardiff laboratory. Therefore, in order to assess the reliability of the effects we report in Experiments 1-3, the next experiment replicated the methodology of Experiment 1 with a different set of odors. In addition, the experiment was designed to examine the role of verbal mediation in serial recognition memory for odors. To this end, the experiment employed a set of seven preselected odors with an average nameability score of $68 \%$. Nameability was assessed in a separate study of 30 participants who were each presented with a sequence of 24 odors. Each odor was presented to the participant for a duration of $5 \mathrm{sec}$. Immediately following each presentation, the participant was required to produce the verbal label typically associated with that odor. The seven odors with the highest correct nameability scores were selected for the experiment. 


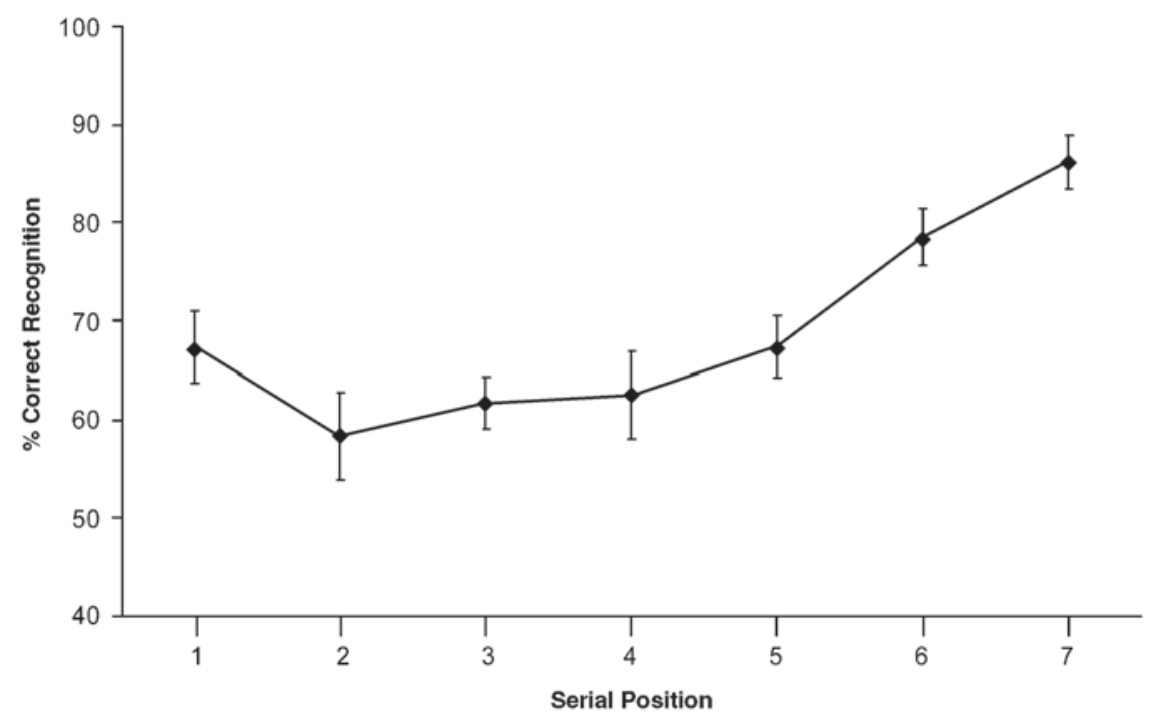

Figure 3. Percentage of odors correctly recognized at each serial position, with standard error bars, for Experiment 3.

It has been argued previously (e.g., White \& Treisman, 1997) that primacy in serial recall of odors might conceivably be due to rehearsal of the verbal label associated with the odor. Although there is some supporting evidence for this suggestion (e.g., Larsson \& Backman, 1997), this seems an unlikely candidate to explain Reed's (2000) pattern of data. As reported by Reed (2000), the mean number of correct explicit verbal labels supplied by participants after completion of Experiment 3 in his series was only $2.8(S D=1.2)$. Nine odors were utilized in that experiment, and this score equates to $31 \%$ correct nameability. Furthermore, additional experiments reported by Reed (2000, Experiments 4 and 5) examined directly the role of articulatory suppression on serial recognition memory for odors. For both experiments, one group of participants was required to repeat the word the rapidly throughout each 3-sec ISI, whereas another group was required to sit quietly throughout each ISI. Results for both experiments exhibited primacy and recency even when articulatory rehearsal was minimized by the concurrent suppression task. Therefore, in Experiment 4, we aimed to assess the potential role of verbal recoding and rehearsal processes more directly. We maximized the participants' opportunity to adopt verbal processing strategies by employing odors with a relatively high name accessibility score. To the extent that verbal recoding and rehearsal processes mediate serial recognition for these stimuli, then primacy in particular should be evident in the serial recognition scores for Experiment 4.

\section{Method}

Participants. Twenty-four psychology undergraduates ( 8 males, 16 females; mean age $=22$ years 4 months) from Cardiff University participated in exchange for course credit. None had participated in the previous experiments.

Materials. The olfactory stimuli (supplied by Dale Air) comprised the seven nameable odors identified previously $(68 \%$ correct nameability in the prestudy). The odors were Lemon, Chocolate, Coffee, Banana, Mixed Herbs, Peppermint, and Liquorice.

Design and Procedure. The design and procedure were the same as those described for Experiment 1.

\section{Results and Discussion}

Figure 4 shows the mean percentage of correct item recognition for each serial position. Visual inspection of Figure 4 suggests no evidence of either primacy or recency, and this was confirmed by both ANOVA with serial position as a repeated measures factor $[F(4,92)=$ $\left.0.819, M S_{\mathrm{e}}=0.69\right]$ and trend analysis in which both the linear and the quadratic components were nonsignificant $[t(23)=0.47$, and $t(23)=0.66$, respectively $]$.

Note that, although the mean correct recognition rate for Experiment 4 (72\%) approximated that for Experiment $1(70 \%)$, the recognition profiles across serial positions were quite different (see Figures 1 and 4). These equivalent overall recognition scores together with the flat recognition profile for the nameable odors (and, in particular, the lack of primacy) mitigates the role of verbal processing in Experiment 4. Although there is no ready explanation for the complete lack of a recency effect in Experiment 4, these data, notwithstanding differences in the stimuli employed, again offer no support to the pattern of data reported by Reed (2000).

\section{EXPERIMENT 5}

In contrast to Experiment 4, the next experiment was designed with the intention of minimizing the role of verbal labeling in odor recognition by employing a set of seven relatively hard-to-name odors. To the extent that verbal labeling is important in this task, it is predicted that the use of this set of odors will reduce overall recognition performance relative to that in the previous experiment. In addition, we predict a complete absence of primacy if this 


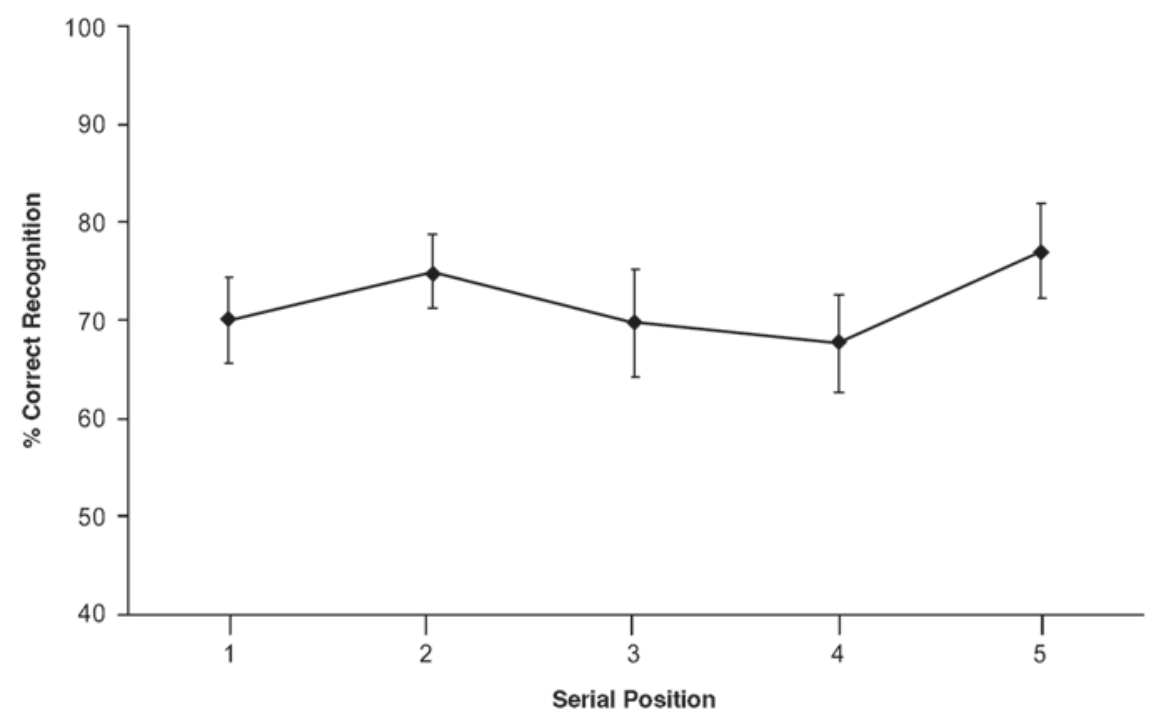

Figure 4. Percentage of odors correctly recognized at each serial position, with standard error bars, for Experiment 4.

component of serial recognition memory is underpinned by verbal rehearsal.

\section{Method}

Participants. Twenty-four psychology undergraduates $(9$ females, 3 males; mean age $=19$ years 4 months) from Cardiff University participated in exchange for course credit. None had participated in the previous experiment.

Materials. The olfactory stimuli (supplied by Dale Air) comprised the seven nonnameable odors. These odors were selected from the prestudy described in Experiment 4 and represented odors with the lowest nameability scores. The odors were Stable/Horses, Coconut, Washday, Gingerbread, Pineapple, Havana Cigar, and Mahogany, and their average nameability score was $23 \%$.
Design and Procedure. The design and procedure were the same as those described for Experiment 1.

\section{Results and Discussion}

Figure 5 shows the mean percentage of correct item recognition for each serial position. Although the pattern of data suggests superior recognition at Serial Positions 4 and 5 relative to that at Serial Position 1, an ANOVA revealed a null effect of serial position $[F(4,92)=1.714$, $\left.M S_{\mathrm{e}}=0.51\right]$. Trend analysis revealed a marginal linear component $[t(23)=2.05, p=.052]$, reflecting the tendency toward recency, and a nonsignificant quadratic component $[t(23)=1.01]$.

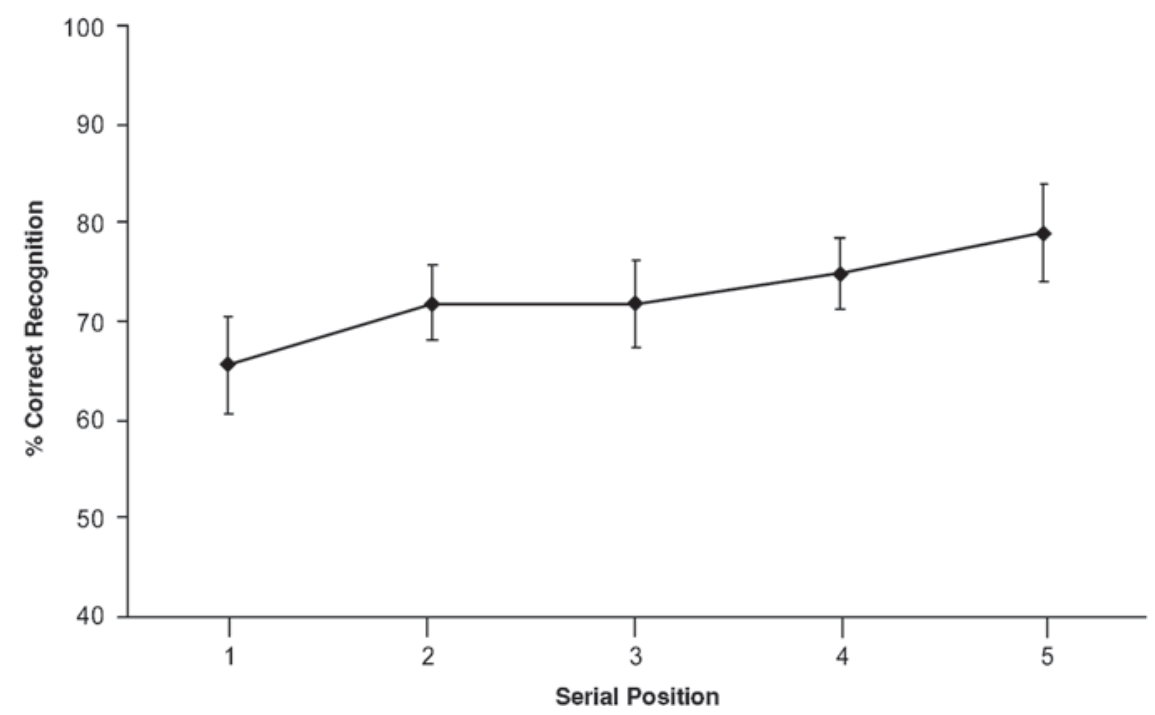

Figure 5. Percentage of odors correctly recognized at each serial position, with standard error bars, for Experiment 5. 
The experiments reported thus far varied in type of stimuli, sequence length, and the duration of stimulus presentation. Nevertheless, the results are similar in two respects: the overall levels of recognition and the absence of primacy. The recency effect was present in Experiments 1,3 , and 5 but was most convincing for sequences of seven odors, as reported in Experiment 3.

\section{EXPERIMENT 6}

One widely used methodology for testing the role of verbal mediation in memory tasks is that of articulatory suppression. The participant is usually required to repeat a word (e.g., "the") throughout the acquisition and/or retention phase of the memory task. Memory performance is typically impaired under this condition in contrast to a silent control condition. The interpretation of such impairment normally assumes that the word repetition task prevents verbal recoding and rehearsal of the to-beremembered target stimuli (e.g., Mahrer \& Miles, 1999; Miles \& Borthwick, 1996). This technique was employed by Reed (2000, Experiments 4 and 5). In his experiments, participants were required to continuously repeat "the" during the 3-sec ISIs for five-item lists (Experiment 4) and four-item lists (Experiment 5). In both experiments, the analyses show that participants' recognition was superior at the start and end of the list (reflecting primacy and recency) and that there was a null effect of articulatory suppression (Experiment 5) and a marginal effect in Experiment 4. The conclusion drawn is that the findings of primacy and recency for serial recognition of odors are not mediated by verbal rehearsal.

The present experiment tested 24 participants, who were each required to complete four blocks of five trials (as in other experiments in this series), in both a quiet condition and an articulatory suppression condition. To facilitate articulatory suppression, we increased the ISI to $5 \mathrm{sec}$. To avoid olfactory fatigue effects (Reed, 2000), we completed the two testing sessions $24 \mathrm{~h}$ apart in a counterbalanced design. Given that the extent that serial recognition of olfactory stimuli is, at least partly, underpinned by a process of verbal mediation, performance should be impaired across all serial positions in the articulatory suppression condition. On the basis of the results of the previous experiments in this series, we predict an absence of primacy in both conditions.

\section{Method}

Participants. Twenty-four psychology undergraduates (14 males, 10 females; mean age $=22$ years 9 months) from Cardiff University participated in exchange for course credit or payment. None had participated in the previous experiments.

Materials. The olfactory stimuli were the same as those described for Experiment 4.

Design and Procedure. A repeated measures design was adopted such that each participant performed the experimental task twice with an interval of approximately $24 \mathrm{~h}$ between each experimental session. Within each experimental session, a repeated measures design as described for Experiment 1 was adopted. The ISI was increased from 2 to $5 \mathrm{sec}$. The two experimental sessions differed in one respect only. For one session, the participant remained silent during each 5-sec ISI. For the other session, the participant was required to repeat "the" continuously and as rapidly as possible throughout each ISI. The order of experimental sessions was counterbalanced across participants. The participants were awarded either course credit or payment upon completion of the second experimental session.

\section{Results and Discussion}

Figure 6 shows the mean percentage of correct item recognition for each serial position in both the quiet condition and the articulatory suppression condition. The recognition data reflect very similar rates of recognition for

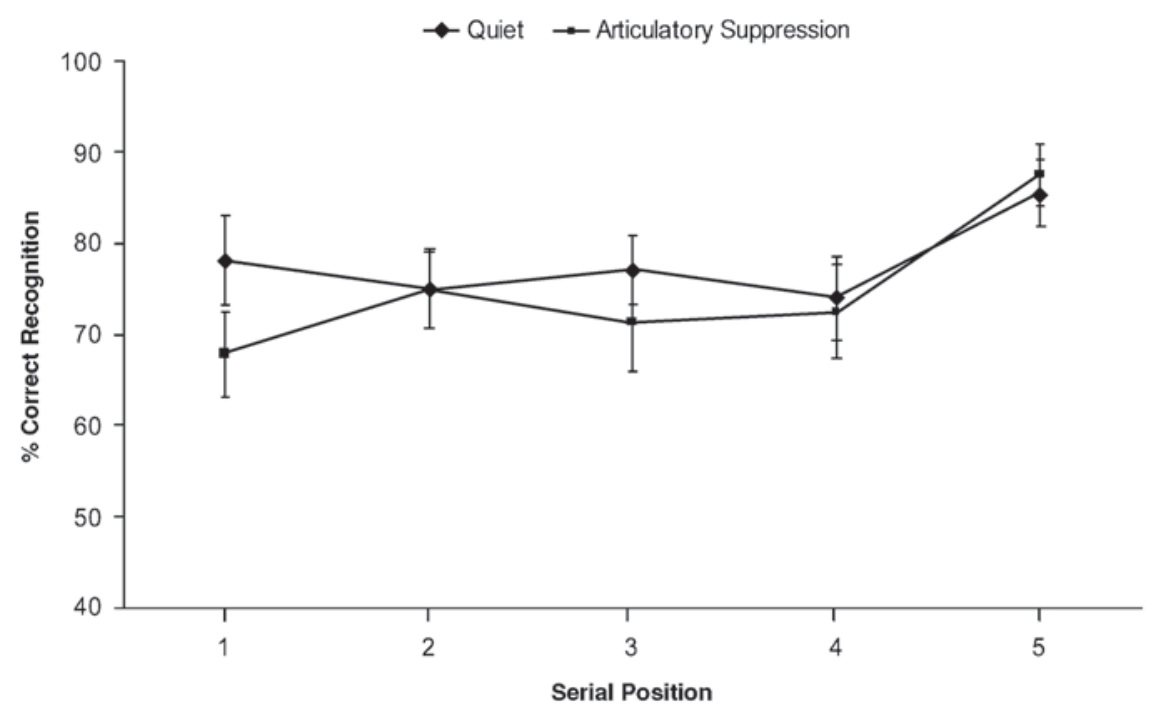

Figure 6. Percentage of odors correctly recognized at each serial position, with standard error bars, for both the quiet and articulatory suppression conditions for Experiment 6. 
Serial Positions 1-4, with superior recognition at Serial Position 5, for both conditions.

This impression was reflected in a two-factor ANOVA with experimental condition and serial position as repeated measures factors that revealed a marginal effect of experimental condition $\left[F(1,23)=4.3, M S_{\mathrm{e}}=0.39\right.$, $p=.049$; means $=79 \%$ and $75 \%$ for the quiet and articulatory suppression conditions, respectively]. The main effect of serial position was significant $[F(4,44)=3.85$, $\left.M S_{\mathrm{e}}=0.71, p<.02\right]$, but the interaction between experimental condition and serial position failed to achieve significance $\left[F(4,92)=1.34, M S_{\mathrm{e}}=0.57\right]$. For the quiet condition, trend analysis revealed both linear and quadratic components to be nonsignificant $[t(23)=1.3$, and $t(23)=1.05$, respectively]. For the articulatory suppression condition, trend analysis revealed a significant linear component $[t(23)=2.7, p<.02]$ and a nonsignificant quadratic component $[t(23)=1.9, p=.069]$.

Again, the results of this experiment are consistent with the majority of those reported earlier in the sequence: that is, a trend toward recency with no evidence of primacy. Consistent with the findings of Reed (2000), the results of this experiment (with extended ISIs) reflected a detrimental, albeit marginal, effect of articulatory suppression on the recognition rates. However, although the interaction between experimental condition and serial position was nonsignificant, Figure 6 clearly shows that the effect of articulatory suppression was not equivalent across all serial positions. Given the marginal and variable effect of articulatory suppression here, no strong statement concerning the effect of this variable can be made. Note, however, that a recency effect is suggested in both experimental conditions.

\section{EXPERIMENT 7}

The present series of experiments has demonstrated that serial recognition memory for a range of olfactory stimuli consistently exceeds that which is predicted by chance $(50 \%)$. This finding persists across a range of list lengths and under conditions of articulatory suppression. The final experiment in the series was designed to reduce recognition performance by interleaving a to-be-ignored odor with the to-be-recognized list items. The logic here is that the interleaved odor will act as a suffix and overwrite the olfactory representation of the list item immediately preceding it. This technique arises from previous research in our laboratory (Miles \& Jenkins, 2000), which has demonstrated that the recency effect in serial recall of odors is attenuated by the introduction of a same modality (i.e., olfactory suffix) but is immune to the effects of a different modality (i.e., auditory suffix). Previous work utilizing the interleaved paradigm with free recall of auditorily presented list items (e.g., Gardiner \& Gregg, 1979) has demonstrated a long-term modality effect, as described first by Bjork and Whitten (1974). That is, interleaving periods of auditory distraction render the final list item invulnerable to interference from subsequent auditory stimulation. In contrast, Hitch (1975, Experiment 2) required serial recall of auditorily presented digits. In the condition in which the suffix was presented after every list item, a suffix effect was observed that was equivalent in magnitude to that observed when the suffix was presented following the final list item only. Greene, Elliot, \& Smith (1988, Experiment 4), in addition to replicating the effect demonstrated by Hitch, showed a significant reduction in recall across all serial positions in the interleaved suffix condition. The effect was replicated using color names rather than digits for the to-be-remembered items (Experiment 5) and using lists of unrelated words. The present experiment was designed to assess the extent to which the effect shown by Greene et al. generalizes to a recognition task employing olfactory stimuli.

\section{Method}

Participants. Twenty-four psychology undergraduates (13 males, 11 females; mean age $=23$ years 5 months) from Cardiff University participated in exchange for course credit or payment. None had participated in the previous experiments.

Materials. The olfactory stimuli were the same as those described for Experiment 4. The interleaved odor was Vanilla.

Design and Procedure. A repeated measures design was adopted such that each participant performed the experimental task twice with an interval of approximately $24 \mathrm{~h}$ between each experimental session. Within each experimental session, a repeated measures design as described for Experiment 1 was adopted. The two experimental sessions differed in one respect only. For one session, the participant remained silent during each 5-sec ISI. For the other session, the participant was required to sniff the Vanilla odor repeatedly throughout each ISI. The order of experimental sessions was counterbalanced across participants. The participants were awarded either course credit or payment on completion of the second experimental session.

\section{Results and Discussion}

Figure 7 shows the mean percentage of correct item recognition for each serial position in both the standard condition and the interleaved condition. A two-factor ANOVA with experimental condition and serial position as repeated measures revealed a null effect of experimental condition $\left[F(1,23)=0.86, M S_{\mathrm{e}}=0.69 ;\right.$ means $=$ $78 \%$ and $75 \%$ for the standard presentation condition and the interleaved suffix condition, respectively], a null effect of serial position $\left[F(4,92)=1.85, M S_{\mathrm{e}}=0.82\right]$, and no interaction $(F=1.07)$. For the standard presentation condition, trend analysis revealed both linear and quadratic components to be nonsignificant $[t(23)=1.07$, and $t(23)=0.8$, respectively]. For the interleaved suffix condition, trend analysis revealed a significant linear component $[t(23)=2.1, p=.044]$, and a nonsignificant quadratic component $[t(23)=1.2]$.

The data from Experiment 7 are consistent with those of all of the previous experiments reported here in their failure to demonstrate a primacy effect. Presentation of a redundant odor as an interleaved suffix did not impair recognition performance relative to the standard presentation condition: a finding that is in contrast to those of Hitch (1975) using serial recall rather than recognition. In addition, the present findings contrast with Walk and 


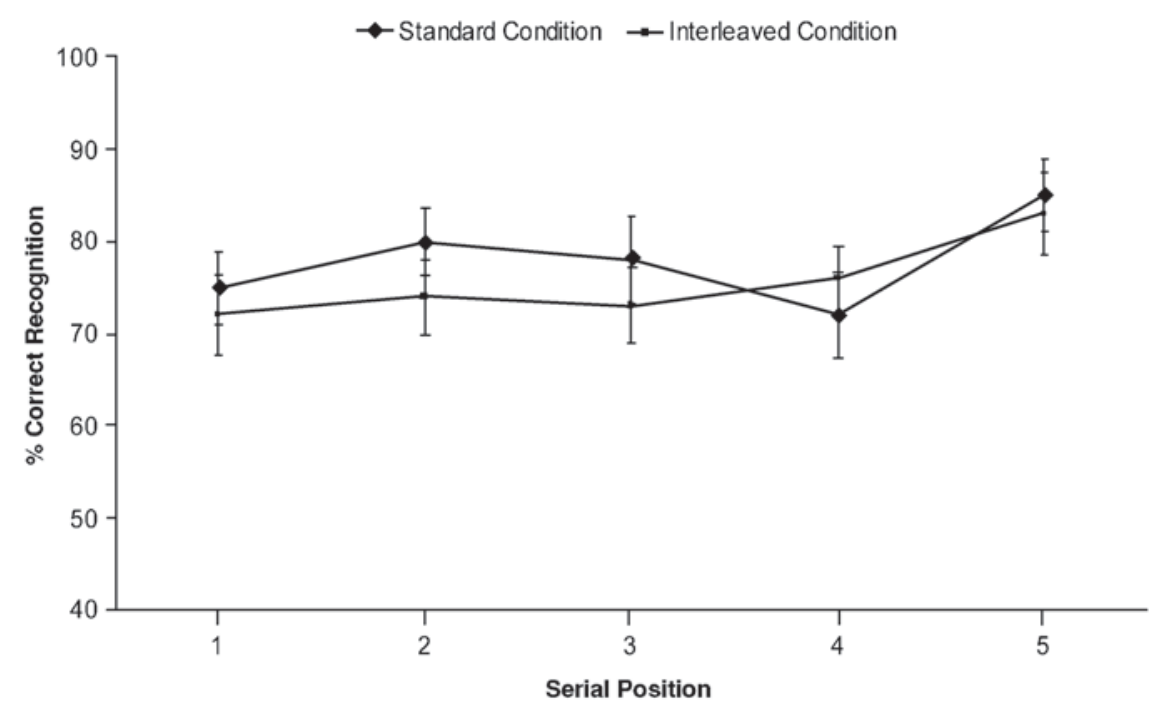

Figure 7. Percentage of odors correctly recognized at each serial position, with standard error bars, for both the standard and interleaved conditions for Experiment 7.

Johns (1984) who, using a four-alternative forced choice procedure, showed that distractor odors interpolated between odor learning and that testing impaired odor recognition. Notwithstanding the variation in retention functions, the data do, however, find support from Jones, Roberts, and Holman (1978), who showed that shortterm odor memory was immune to the effects of odors intervening between learning and testing. The data with respect to the representation of odors within short-term memory therefore remain equivocal. This is largely due to differences in testing technique. The argument that odors are represented in memory as unitary, distinctive events with minimal attribute redundancy (Engen, 1987; Lawless, 1978), which render them immune to interference from similar stimuli, gains some support from these data. What does not appear to be in empirical doubt from the present series of experiments is that the recognition rate for a range of odors, using a $2 \mathrm{AFC}$ task with immediate testing, remains consistently at around 70\%. With the exception of a marginal finding in Experiment 3, the pattern of recognition shows no evidence of primacy. Recency is statistically evident in some of the experiments, and a trend is evident in others. This pattern of findings is consistent with the body of work examining recency with short retention intervals in recognition for hard-to-name, visual stimuli (e.g., Kerr et al., 1999; Neath, 1993; Phillips \& Christie, 1977).

\section{GENERAL DISCUSSION}

The question that motivated this series of experiments focused on the extent to which the effects of primacy and recency in immediate serial recognition for odor sequences, as reported by Reed (2000), are reliable. Previous recognition studies employing hard-to-name visual stimuli (e.g., Kerr et al., 1999; Neath, 1993; Phillips \& Christie, 1977) with immediate testing consistently dem- onstrate recency in the absence of primacy. Theoretically, therefore, one might not predict the effects shown by Reed (2000). However, there are slight differences in the details of the recognition tasks employed by these authors and those employed by Reed (2000) and ourselves. The earlier studies present a set of unique stimuli in each sequence followed by a pair of test stimuli from which the participant is required to report which of the two stimuli is familiar. Thus, the participant's judgment relies on a "pure" measure of familiarity, in the sense that such judgment is not contaminated by earlier presentations of the stimulus. The experiments reported both here and by Reed employed a limited set of stimuli such that each stimulus was utilized a number of times throughout the experiment. The memory sequence was followed by two test stimuli, only one of which was in the preceding sequence. However, the distractor stimulus would have been familiar to the participant from earlier trials, and, therefore, the participant was making a judgment about the relative familiarity of one of the two test stimuli. Notwithstanding this difference in test procedures, it is, nevertheless, difficult to develop an argument whereby this difference in procedure should impact on the primacy component of the serial position curve.

An important methodological variable that positively influences primacy while simultaneously producing negative recency in recognition tasks is the retention interval between sequence presentation and the recognition task (Knoedler, Hellwig, \& Neath, 1999; Korsnes, 1995; Neath, 1993; Neath \& Knoedler, 1994; Wright, Santiago, Sands, Kendrick, \& Cook, 1985). Consider the experiments reported by Neath (1993). Participants were presented with sequences of digitized photographs of snowflakes followed by a recognition test after retention intervals of varying duration. For both between- and within-subjects designs, as the retention interval increased, primacy increased from chance to reliably better than chance. In 
contrast, recency, which was reliable in the immediate test condition, decreased to chance levels as the retention interval increased. Furthermore, overall accuracy rates were equivalent across the retention intervals. Work by Knoedler et al. (1999) has demonstrated that the recencyto-primacy shift with increasing delay is maintained when the physical form of the study item and the test item differs (ruling out visual memory) and when only one serial position is tested (ruling out strategy changes). That the recency-to-primacy shift is not species dependent has been shown by Wright et al. (1985), who used a same-different recognition test for sequences of four items employing pigeons, monkeys, and human participants. Recency was reliable in the immediate $(0 \mathrm{sec})$ retention interval condition for all participant groups. However, as the retention interval increased (to $10 \mathrm{sec}$ for the pigeons, $30 \mathrm{sec}$ for the monkeys, and $100 \mathrm{sec}$ for the humans), recency was no longer apparent, but primacy was reliable. As argued by Wright (1994), the primacy effect in nonverbal recognition tasks appears to be dynamic in nature. His retentioninterval experiments demonstrate that the U-shaped serial position function represents a transitional state between an all-recency and an all-primacy function.

On possible mechanism underpinning the transition from recency to primacy relies on the temporal distinctiveness of sequence items, as proposed originally by Murdock (1960) and modeled formally by Neath (1993). A temporal distinctiveness account can handle the traditional bow-shaped serial position curve on the premise that the most recent items in a uniformly spaced list enjoy greater temporal distinctiveness than do items in the middle of the list, and, similarly, the early list items are temporally more distinct than are the middle list items. As the retention interval increases, the most recent items fade from short-term memory producing negative recency. However, earlier items enjoy relative stability in long-term memory, and the recognition profile reflects the temporal distinctiveness of those items and therefore produces primacy.

More recent evidence, however, has failed to confirm the recency-to-primacy shift. For instance, Kerr et al. (1999) reported a series of experiments in which participants were presented with a series of four novel visual items (visual patterns and faces), followed by both immediate and delayed (5 and $10 \mathrm{sec}$ ) single-probe recognition tests. Delaying the recognition test consistently reduced final-item recognition (recency), but in only one experiment was this associated with an increase in primacy-a finding they failed to replicate. Kerr et al. (1998) demonstrated the recency-to-primacy shift when participants were presented with a sequence of four unfamiliar faces and were required to state the serial position of a probe face after 0 or $10 \mathrm{sec}$. However, analysis of the distribution of responses showed a change in response bias with retention interval. Correcting for this bias eliminated the recency-to-primacy shift. Note, however, this bias was apparent in a task in which participants were required to state explicitly the serial position of the test item. Whether such a bias is evident in the more conventional yes-no methodology (which requires a familiarity judgment rather than a positional judgment) remains an open question.

In both the present experiments and those reported by Reed (2000), the recognition test followed a short retention interval of $3 \mathrm{sec}$. On the basis of Wright et al.'s. (1985) observation that, with human participants, (1) recency was evident only after a 2 -sec retention interval, (2) the full serial position curve was evident after a 10 -sec retention interval, and (3) primacy coupled with negative recency was evident after a 100 -sec retention interval, there is no strong evidence to predict either a full serial position curve or primacy only in these studies. However, Wright et al.'s observations were based on sequences of kaleidoscope patterns and therefore tested visual memory. It remains to be established whether or not similar temporal parameters govern the recency-to-primacy shift for olfactory stimuli.

Recency should always be enhanced in recognition studies employing reverse testing, because the same (final list) stimulus is re-presented in immediate succession and that stimulus has not been presented in earlier trials. Indeed, we have used this procedure in supplementary work in our own laboratory. Johnson, Miles, and Beacham (2005) presented each of 24 participants with a series of trials in which each trial comprised the presentation of a sequence of six unique odors followed immediately by a series of 2AFC tasks. With reverse testing of the odor sequence, where participants were presented with a series of paired alternatives at test that tracked backward through the sequence, primacy was absent (63\% correct recognition at Serial Position 1), but recency was evident ( $82 \%$ correct recognition at Serial Position 6). In contrast, forward testing produced a flat recognition profile, with recognition rates of $70 \%$ and $69 \%$ for Serial Positions 1 and 6 , respectively. Forward testing maximizes the probability of producing a primacy effect because, for each sequence, the first recognition test involves a re-presentation of the first odor in the sequence. However, our data provide no evidence of primacy. Thus, using a modified recognition procedure that follows very closely that reported by Kerr et al. (1999), the pattern of results for both odors and unfamiliar faces is the same: pronounced recency in the absence of primacy with backward testing and the absence of each of these with forward testing.

In conclusion, precisely why the findings in the present series diverged consistently from those reported by Reed (2000), particularly for the experiments that replicated precisely his methodology, remains unclear. However, given the range of stimuli and manipulations reported here and the consistency of the findings with respect to extant theory, we suggest that immediate serial recognition of memory for odors using a $2 \mathrm{AFC}$ recognition task does not produce a full serial position curve. In particular, the consistent failure to demonstrate primacy (with the exception of the marginal finding in Experiment 3) suggests that the results reported by Reed (2000) are not easily replicable and may be artifactual in their origin. 


\section{REFERENCES}

Annett, J. A., \& Lorimer, A. W. (1995). Primacy and recency in recognition of odors and recall of odor names. Perceptual \& Motor Skills, 81, 787-794.

Avons, S. E., WARD, G., \& Melling, L. (2004). Item and order memory for novel visual patterns assessed by two-choice recognition. Quarterly Journal of Experimental Psychology, 57A, 865-891.

BJork, R. A., \& Whitten, W. B. (1974). Recency-sensitive retrieval processes. Cognitive Psychology, 6, 173-189.

Engen, T. (1987). Remembering odors and their names. American Scientist, 75, 497-503.

Gaffan, E. A. (1992). Primacy, recency, and the variability of data in studies of animals' working memory. Animal Learning \& Behavior, 20, 240-252.

GafFan, E. A. (1994). Primacy in animals' working memory: Artifacts. Animal Learning \& Behavior, 22, 231-232.

GAFFAN, E. A., \& GAFFAN, D. (1992). Less-than-expected variability in evidence for primacy and von Restorff effects in rats' nonspatial memory. Journal of Experimental Psychology: Animal Behavior Processes, 18, 298-301.

Gardiner, J. M., \& GregG, V. H. (1979). When auditory memory is not overwritten. Journal of Verbal Learning \& Verbal Behavior, 18, 705-719.

Greene, R. L., Elliot, C. L., \& Smith, M. D. (1988). When do interleaved suffixes improve recall? Journal of Memory \& Language, 27, $560-571$.

Herz, R., \& Engen, T. (1996). Odor memory: Review and analysis. Psychonomic Bulletin \& Review, 3, 300-313.

Hitch, G. J. (1975). The role of attention in visual and auditory suffix effects. Memory \& Cognition, 3, 501-505

Johnson, A., Miles, C., \& Beacham, H. A. (2005). Item and order memory for odours assessed by two-choice recognition. Manuscript in preparation.

Jones, F. N., Roberts, K., \& Holman, E. (1978). Similarity judgments and recognition memory for common spices. Perception \& Psychophysics, 24, 2-6.

KAISER, L., \& DE Jong, R. (1993). Multi-odour memory influenced by learning order. Behavioural Processes, 30, 175-184.

Kerr, J. R., Avons, S. E., \& WARD, G. (1999). The effect of retention interval on serial position curves for item recognition of visual patterns and faces. Journal of Experimental Psychology: Learning, Memory, \& Cognition, 25, 1475-1494.

Kerr, J. R., WARD, G., \& Avons, S. E. (1998). Response bias in visual serial order memory. Journal of Experimental Psychology: Learning, Memory, \& Cognition, 24, 1316-1323.

Kesner, R. P., Chiba, A. A., \& JaCKson-Smith, P. (1994). Rats do show primacy and recency effects in memory for lists of spatial locations: A reply to Gaffan. Animal Learning \& Behavior, 22, 214-218.

Knoedler, A. J., Hellwig, K. A., \& Neath, I. (1999). The shift from recency to primacy with increasing delay. Journal of Experimental Psychology: Learning, Memory, \& Cognition, 25, 474-487.

Korsnes, M. S. (1995). Retention intervals and serial list memory. Perceptual \& Motor Skills, 80, 723-731.

LARSSON, M., \& BACKMAN, L. (1997). Age-related differences in episodic odour recognition: The role of access to specific odour names. Memory, 5, 361-378.

LAWLESS, H. T. (1978). Recognition of common odors, pictures, and simple shapes. Perception \& Psychophysics, 24, 493-495.

Mahrer, P., \& Miles, C. (1999). Memorial and strategic determinants of tactile recency. Journal of Experimental Psychology: Learning, Memory, \& Cognition, 25, 630-643.

Miles, C., \& BorThwick, H. (1996). Tactile short-term memory revisited. Memory, 4, 655-668.

Miles, C., \& Jenkins, R. (2000). Recency and suffix effects with immediate recall of olfactory stimuli. Memory, 8, 195-206.

Murdock, B. B., Jr. (1960). The distinctiveness of stimuli. Psychological Review, 67, 16-31.

NeAth, I. (1993). Distinctiveness and serial position effects in recognition. Memory \& Cognition, 21, 689-698.

Neath, I., \& Knoedler, A. J. (1994). Distinctiveness and serial position effects in recognition and sentence processing. Journal of Memory \& Language, 33, 776-795.

Phillips, W. A., \& Christie, D. F. M. (1977). Components of visual memory. Quarterly Journal of Experimental Psychology, 29, 117 133

Rawlins, J. N. P., Deacon, M. R. J., Tai, C. T., \& Aggleton, J. P. (1992). Doubts concerning primacy in rats' nonspatial recognition memory: Reply to Gaffan and Gaffan. Journal of Experimental Psychology: Animal Behavior Processes, 18, 302-304.

REED, P. (1992). Fewer doubts concerning rats' serial position performance: Reply to Gaffan and Gaffan (1992) and Rawlins, Deacon, Chih-Ta, and Aggleton (1992). Journal of Experimental Psychology: Animal Behavior Processes, 18, 305-307.

REED, P. (1994). Less than expected variance in studies of serial position effects is not a sufficient reason for caution. Animal Learning \& Behavior, 22, 224-230.

REED, P. (2000). Serial position effects in recognition memory for odors. Journal of Experimental Psychology: Learning, Memory, \& Cognition, 26, 411-422.

Reed, P., Chin-Ta, T., Aggleton, J. P., \& Rawlins, J. N. P. (1991). Primacy, recency and the von Restorff effect in rats' nonspatial recognition memory. Journal of Experimental Psychology: Animal Behavior Processes, 17, 36-44.

Surprenant, A. M., Pitt, M. A., \& Crowder, R. G. (1993). Auditory recency in immediate memory. Quarterly Journal of Experimental Psychology, 46A, 193-223.

WALK, H. A., \& JoHns, E. E. (1984). Interference and facilitation in short-term memory for odors. Perception \& Psychophysics, 36, 508514

Ward, G., Avons, S. E., \& Melling, L. (2005). Serial position curves in short-term memory: Functional equivalence across modalities. Memory, 13, 308-317.

White, T. L. (1992, March). A comparison of item and order processing in olfactory and verbal short-term memory. Paper presented at the fourth annual AchemS conference, Sarasota, FL.

White, T. L. (1998). Olfactory memory: The long and the short of it Chemical Senses, 23, 433-441.

White, T. L., \& Treisman, M. (1997). A comparison of the encoding of content and order in olfactory memory and in memory for visually presented verbal materials. British Journal of Psychology, 88, 459-472.

Wright, A. A. (1994). Primacy effects in animal memory and human nonverbal memory. Animal Learning \& Behavior, 22, 219-223.

Wright, A. A., Santiago, H. C., Sands, S. F., Kendrick, D. F., \& Cook, R. G. (1985). Memory processing of serial lists by pigeons, monkeys, and people. Science, 229, 287-289.

(Manuscript received February 19, 2004 revision accepted for publication November 3, 2004.) 